\title{
Neuroplastic Effect of Constraint-Induced Music Therapy on Hearing Recovery in Patients with Sudden Sensorineural Hearing Loss
}

\author{
Chin-Lung Kuo, MD, PhD ${ }^{1,2^{*}}$
}

' Institute of Brain Science, National Yang-Ming University School of Medicine, Taipei, Taiwan

2Department of Otolaryngology-Head and Neck Surgery, Taoyuan Armed Forces General Hospital, Taoyuan, Taiwan

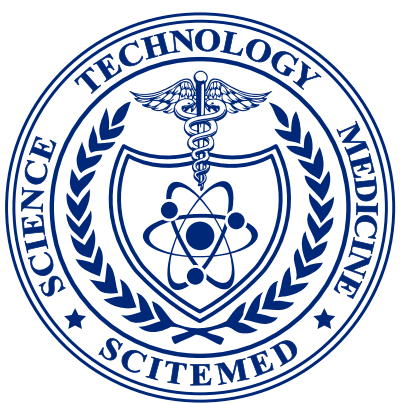

\begin{abstract}
Objective: Constraint-induced music therapy (CIMT) has been shown to enhance hearing recovery in patients with sudden sensorineural hearing loss (SSNHL) by preventing maladaptive reorganization of the auditory cortex. Our objective in this study was to assess the effectiveness of CIMT in restoring hearing among patients with SSNHL.

Methods: The study included prospective (CIMT group) and retrospective (non-CIMT group) study arms. CIMT is characterized by (1) plugging the healthy ear to induce temporary artificial hearing loss (i.e., constraint) and (2) simultaneous acoustic stimulation of the affected ear using relaxing music. The outcome variables used to evaluate hearing recovery included (1) hearing threshold, (2) interaural hearing gap, and (3) hearing recovery rate. We measured the P300 component of long-latency auditory evoked potential to analyze brain activity to determine the appearance of neuroplasticity in assessing a subgroup of six patients with CIMT. All of the patients in the study also received conventional steroid therapy.

Results: The CIMT and non-CIMT groups were comparable in terms of pre-treatment hearing level $(P=0.710)$, age $(P=0.124)$, gender $(P=0.272)$, and side of hearing loss $(P=0.132)$. In both groups, we observed a significant improvement in hearing thresholds at two weeks and four weeks after treatment (all $P<0.01)$. Nevertheless, we did not observe a statistically significant difference in hearing recovery when using hearing threshold, interaural hearing gap, or hearing recovering rate as outcome variables $(P>0.05)$. This observation was verified using multivariate analysis (non-CIMT vs. CIMT, odds ratio 3.84 , $95 \%$ confidence interval $0.18-81.65, P=0.388$ at two weeks after treatment; odds ratio $2.70,95 \%$ confidence interval $0.15-47.60, P=0.497$ at four weeks after treatment). $\mathrm{P} 300$ measurements conducted on affected ears failed to identify significant signs of neuroplastic change in response to CIMT (P $=0.063$ for the amplitude comparison; $P=0.094$ for the latency comparison).

Conclusion: CIMT is a safe, cost-effective addition to corticosteroid treatment for SSNHL patients. It is also possible that CIMT provides an enjoyable therapeutic adjunct for the relief of stress and anxiety associated with SSNHL. However, our results failed to identify the additive effect of CIMT on hearing recovery in patients with SSNHL. Our study was also unable to confirm the degree of neuroplasticity in patients with CIMT.
\end{abstract}

\section{INTRODUCTION}

Sudden sensorineural hearing loss (SSNHL) refers to a subjective sensation of hearing impairment over a period of less than 72 hours. This condition is typified by specific audiometric criteria: (1) a decrease in hearing of $\geq 30$ decibels $(\mathrm{dB})$, compared to threshold of the premorbid or opposite ear (if premorbid audiometry is unavailable); and (2) hearing loss that affects at least three consecutive frequencies [1]. SSNHL is an otologic emergency, which can be a highly distressing experience for patients, and particularly for those who depend on their hearing for work (e.g., musicians, professional drivers, or athletes). SSNHL can have a tremendous impact on one's quality of life and is often indicative of an elevated risk of adverse cognitive and functional outcomes. In the United States, the number of new cases each year has been estimated at 4,000 [2]. A population-based cross-sectional investigation of SSNHL epidemiology in Germany revealed an incidence of 160 cases of SSNHL per year per 100,000 inhabitants [3]. The high incidence of SSNHL is reflected in the fact that the World Health Organization and the European Union do not consider it a rare disease (prevalence less than 50 per 100,000 inhabitants) [3]. SSNHL typically occurs in middle adulthood and the incidence increases with age [4-6]. Males and females are equally affected [4].

SSNHL can be attributed to an abnormality of the auditory nerve or higher aspects of central auditory perception or processing [1]; however, the cochlea is generally considered the most probable lesion site [7]. The fact is that only $10 \%$ of SSNHL cases can be identified, the most pressing of which are acoustic neuroma, stroke, and malignancy [8]. The remaining $90 \%$ are idiopathic, presumptively attributed to vascular, infectious, immunologic, or multiple etiologies [1]. This lack of clarity regarding etiology has led to the development of various therapy modalities, including systemic and intratympanic steroids, antiviral agents, anticoagulants, volume expanders, vasoactive substances, antioxidants, hyperbaric oxygen, anti-anxiety medication, diuretics (alone or in combination) [1], or observation alone [9]. Among the various treatment modalities, a tapering course of steroids has been widely adopted as the principal treatment for idiopathic SSNHL, with a reported success rate of 50 to $80 \%[4,10,11]$. Nevertheless, several systematic reviews of randomized controlled trials have failed to determine the role of corticosteroid treatment for SSNHL, with conflicting outcomes [1,12-16]. Furthermore, many patients are not candidates for these substances, due to potentially severe side effects, including the suppression of hypothalamic-pituitary-adrenal function, insomnia, weight gain, gastritis, mood changes, hyperglycemia, hypertension, cataracts, opportunistic infections, osteoporosis, and osteonecrosis $[1,17]$. There is a lack of adequately powered randomized trials to support the clinical benefits of other treatment options [1].

Thus, the focus of research on SSNHL has shifted toward neuroplasticitytargeted intervention [18]. Neuroplasticity (i.e., neural plasticity) refers to the ability of the nervous system to reorganize its structure, function, and connections in response to environmental stimuli or demands [19]. It was not until the late 1960s that Raisman introduced the term "neuronal plasticity" to describe a permanent change of the neuropil in the septal nuclei of adult rats in response to original deafferentation. Decades of research have now shown 


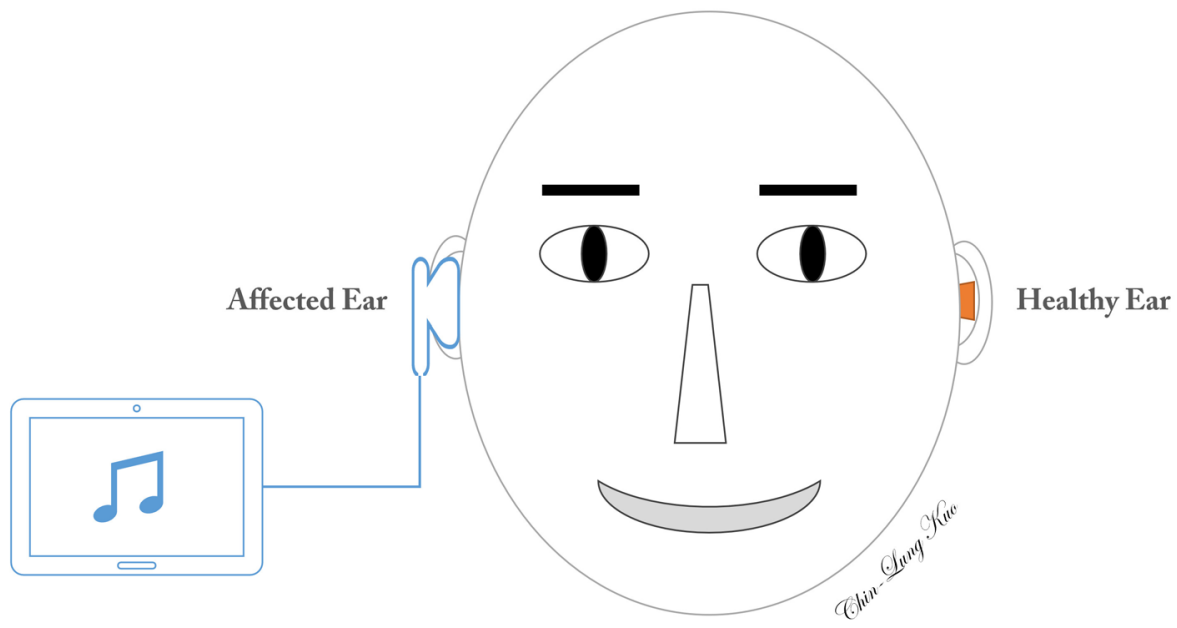

Figure 1. Schematic illustration of constraint-induced music therapy. Music is presented to the affected ear, while the ear canal of the unaffected side is plugged to prevent the admission of sound waves.

that the brain is capable of responding dynamically to a variety of internal and external stimuli [20]. Recent studies have also indicated that neuroplasticity is a fundamental lifelong property of the nervous system [19,21]. In a recent study by Okamoto et al. [22], constraint-induced sound therapy was used to treat patients with idiopathic SSNHL to eliminate or reduce the effects of maladaptive cortical reorganization. The biological plausibility of this approach is based on a neuro-rehabilitation approach and a large body of animal experiments [23-27]. The concept of constraint-induced therapy has previously been used in stroke rehabilitation [28-34]. This involved having hemiplegic patients use their affected limbs while preventing them from using the healthy counterpart by imposing physical constraints. Okamoto et al. also applied this approach to the neuro-rehabilitation of patients with SSNHL. They reported that patients who received constraint-induced sound therapy in conjunction with standard corticosteroid therapy enjoyed significantly better hearing recovery than did those who received only corticosteroid treatments. They attributed those results to brain activity preventing maladaptive reorganization of the auditory cortex.

Nevertheless, there is a lack of clinical evidence to support assertions pertaining to the neurophysiological implications of neuroprotective acoustic training in patients with idiopathic SSNHL. To the best of our knowledge, this was only the second study to provide scientific evidence related to the neuroplasticity of the central auditory nervous system in response to constraint-induced acoustic rehabilitation. Our objective in this study was to assess (1) the additive effect of neuroplasticity on hearing recovery and (2) the unique capacity of constraint-induced acoustic stimulation to prevent maladaptive cortical plasticity in patients with idiopathic SSNHL.

\section{METHODS}

\section{Ethical Considerations}

One arm of this study involved the prospective collection of data between March 1, 2018, and November 1, 2018 (8 months), during which subjects received conventional steroid treatment as well as music therapy. We also con- ducted a retrospective collection of data for the period between April 1, 2016, and August 1, 2018 (2 years), during which subjects received only conventional steroid therapy. The study was conducted in accordance with the Declaration of Helsinki. The prospective study protocol was approved by the Institutional Review Board of Tri-Service General Hospital (No. 1-106-05-189). The purpose, procedures, potential risks, and benefits of the study were thoroughly explained to all candidates, and written informed consent was obtained from all patients prior to participation.

\section{Subjects}

\section{Inclusion criteria}

We specified predefined inclusion and exclusion criteria for participation in the study to minimize potential complications associated with interpretation. Eligible patients were recruited from the Department of Otolaryngology-Head and Neck Surgery, Taoyuan Armed Forces General Hospital, Taiwan.

Enrollment of eligible patients was based on a diagnosis using the following predefined inclusion criteria: (1) unilateral idiopathic sensorineural hearing loss of $\geq 30 \mathrm{~dB}$, which is related to the threshold of the premorbid or opposite ear (in cases where premorbid audiometry was unavailable, we assumed that the audiogram of the unaffected ear is similar to the pre-SSNHL audiogram of the affected ear); (2) sensorineural hearing loss affecting at least three consecutive frequencies; and (3) $\leq 5$ days since symptom onset [22].

\section{Exclusion criteria}

Participants were excluded if they met any one of the following criteria: (1) age $<20$ and $>70$ years; (2) previous or family history of SSNHL; (3) neurological, psychiatric, or cognitive deficits; (4) history of head trauma; (5) air-bone gap of the affected ear, regardless of whether it was associated with a disorder of the middle ear or outer ear; (6) absolute or relative contraindication for steroids, such as a documented allergy to any steroid [35], diabetes with poor control [36,37], hypertension with poor control [37], gastrointestinal ulcerations [37], and a tendency for acne [38]; (7) we also excluded patients who refused to undergo music therapy in the prospective study arm; and (8) those with retro-cochlear causes for SSNHL as evidenced by abnormal differences in inter- 


\begin{tabular}{|c|c|c|c|c|}
\hline Variables & Number (\%) & CIMT & Non-CIMT & $P$ \\
\hline $\begin{array}{l}\text { Pre-treatment } \\
\text { Hearing Level }\end{array}$ & & & & 0.710 \\
\hline$\leq 60 \mathrm{~dB}$ & $19(43)$ & 3 & 16 & \\
\hline$>60 \mathrm{~dB}$ & $25(57)$ & 6 & 19 & \\
\hline Age & & & & 0.124 \\
\hline$\leq 50$ years & $16(36)$ & 1 & 15 & \\
\hline$>50$ years & $28(64)$ & 8 & 20 & \\
\hline Gender & & & & 0.272 \\
\hline Male & $23(52)$ & 3 & 20 & \\
\hline Female & $21(48)$ & 6 & 15 & \\
\hline Side of Ear & & & & 0.132 \\
\hline Right & $22(50)$ & 7 & 15 & \\
\hline Left & $22(50)$ & 2 & 20 & \\
\hline
\end{tabular}

$\mathrm{CIMT}$, constraint-induced music therapy; $\mathrm{dB}$, decibels.

aural latency in wave $\mathrm{V}$ of auditory brainstem responses (ABRs) and magnetic resonance imaging of the brain [39].

\section{Treatment Protocols}

\section{Steroid therapy}

All of the eligible patients (in both study arms) were admitted to the hospital to receive a course of corticosteroids over a period of 7 days ( $1 \mathrm{mg} / \mathrm{kg} / \mathrm{day})$, the dosage of which was subsequently tapered over a period of 7 days $(0.5 \mathrm{mg} / \mathrm{kg} /$ day for 4 days and $0.25 \mathrm{mg} / \mathrm{kg} /$ day for 3 days).

\section{Constraint-induced music therapy (Figure 1)}

In the prospective study arm, constraint-induced music therapy (CIMT) included two major components: (1) plugging the intact ear with a foam ear plug to avoid mechanical acoustic input to the cochlea of the healthy ear, thereby constraining afferent electrical neural impulses on the contralateral auditory cortex; and (2) the simultaneous stimulation of the affected ear in an attempt to prevent maladaptive auditory cortical plasticity on the healthy side [22]. The equipment used to administer CIMT included a music player installed on a tablet (Asus ZenPad 10 Z301 Series, ASUSTeK Computer Inc., Taiwan), a pair of headphones (E-books S39 Gaming Volume Control Hook Headset, Chung Ching Technical Co., Ltd, Taichung, Taiwan), and a foam ear plug (3M 1100, $3 \mathrm{M}$ Co., Minnesota, USA). To enhance the motivation of patients to engage in the music program, we applied relaxing music rather than pure tones or noise [40]. Patients eligible for CIMT underwent a daily six-hour music program (2 hours in the morning, afternoon, and evening), every day for a period of four weeks. Patients adjusted the volume in one of two ways: (1) to a level at which the music sounded as similar as possible to the sounds before SSNHL [22], or (2) to a level that felt most comfortable to the healthy ear. The sound level was limited to $80 \mathrm{~dB}$ [41]. Volume adjustment was meant to increase sound levels in the range of frequencies affected by hearing loss and to avoid potential complications associated with loudness.

\section{Measurements}

\section{Audiometric examinations}

All participants underwent pure tone audiometry (PTA) examinations using an audiometer (Audiometer GSI-68, Grason-Stadler Inc., Minnesota, USA) to determine the air and bone conduction thresholds of affected and unaffected ears, using a step size of $5 \mathrm{~dB}$ at frequencies between $250 \mathrm{~Hz}$ and $8000 \mathrm{~Hz}$, in accordance with the modified Hughson-Westlake procedure [42]. Data were compiled on pre- and post-treatment PTA with mean thresholds determined at $0.5,1,2$, and $3 \mathrm{kHz}$. The hearing thresholds of both ears were measured every day during the hospital stay to monitor recovery. Hearing thresholds of affected and unaffected ears were also measured when the patients came to our outpatient department for follow-up one week and three weeks after discharge.

\section{Neuroplasticity measurements: auditory evoked potential (P300)}

The P300 component of long-latency auditory evoked potential was captured using an evoked potential system (Intelligent Hearing Systems Smart-EP, Intelligent Hearing Systems Co., Florida, USA) for the measurement of neuroplasticity. The skin was cleaned with abrasive paste before attaching electrodes to the skin with electrolytic paste and adhesive tape. P300 was recorded using the oddball paradigm, in which two tone burst stimuli were presented in a random order. One of the two stimuli (standard stimulus, $1000 \mathrm{~Hz}$ ) was presented more than the other (deviant stimulus, $2000 \mathrm{~Hz}$ ). These monaural stimuli were delivered to the affected or the healthy ear for 70 ms at 80-90 $\mathrm{dBnHL}$ (standard $90 \mathrm{dBnHL}$ and target $80 \mathrm{dBnHL}$ ) at a presentation speed of 1.1 stimuli per second using an exact Blackman envelope. The participants were instructed to count the deviant stimulus, which was presented with probability of $15 \%$. P300 was recorded using four electrodes placed at Fz (active electrode), Fpz (ground electrode), and $\mathrm{M} 1$ and $\mathrm{M} 2$ (mastoids, reference electrodes). The total number of stimuli ranged between 450 and 550. Responses were collected using impedance values below 5 kohms with band-pass filters of 1 to $30 \mathrm{~Hz}$.

\section{Data Analysis and Statistics}

For both the CIMT and non-CIMT groups, differences in mean hearing threshold were measured in both ears across all measured frequencies during all examinations; i.e., at the time of admission and at two weeks and four weeks after admission. Paired samples t-tests were used to determine the differences between pre- and post-treatment hearing thresholds in order to assess the degree of hearing recovery.

The variables used to evaluate hearing recovery included the following: (1) hearing threshold; (2) interaural hearing gap (i.e., difference in mean hearing threshold between ears) [22]; and (3) hearing recovering rate $=$ (pre-treatment air conduction hearing threshold of the affected ear - post-treatment air conduction hearing threshold of the affected ear) / (pre-treatment air conduction hearing threshold of the affected ear - air conduction hearing threshold of the intact ear), under the assumption that the audiogram of the intact ear was similar to the audiogram of the affected ear prior to the occurrence of SSNHL $[22,41]$.

A Pearson's chi-square test or Fisher's exact test was used when analyzing categorical variables. Multivariate logistic regression was used to identify the factors that affect hearing recovery. Complete hearing recovery was defined as a hearing threshold of $\leq 25 \mathrm{~dB}$ (normal hearing). Factors used as predictors of hearing recovery included age, gender, side of ear, pre-treatment hearing level, and administration of CIMT.

P300 analysis for the CIMT group was based on the presence/absence of responses for each ear. We calculated the mean latencies (millisecond, ms) and amplitudes (microvolt, $\mu \mathrm{V}$ ) in each ear before treatment and four weeks after treatment. A paired sample t-test was used to determine the difference between pre- and post-treatment mean latencies (ms) and amplitudes $(\mu \mathrm{V})$ in both ears in order to evaluate the degree of neuroplasticity.

All statistical analysis was performed using the commercially available software package SPSS, version 18.0 (SPSS, Inc., Chicago, IL, USA), and p values of less than 0.05 were considered statistically significant.

\section{RESULTS}

Patient characteristics are listed in Table 1. Among the 44 patients included in the study, 9 patients were included in the prospective CIMT study arm and 35 patients in the retrospective non-CIMT study arm. The 9 patients in the prospective CIMT group included 6 females $/ 3$ males, involving 7 right ears and 


\section{ORIGINAL}

Table 2. Intra-Group Comparison of Hearing Thresholds at Various Time Points

\begin{tabular}{|c|c|c|c|c|c|c|c|c|}
\hline \multirow[t]{3}{*}{ Time Point } & \multicolumn{4}{|l|}{ CIMT Group (n=9) } & \multicolumn{4}{|c|}{ Non-CIMT Group $(n=35)$} \\
\hline & \multirow{2}{*}{$\begin{array}{l}\text { Hearing Level } \\
(\mathrm{dB}, \text { mean } \pm \mathrm{SD})\end{array}$} & \multicolumn{3}{|c|}{ P Values } & \multirow{2}{*}{$\begin{array}{l}\text { Hearing Level } \\
(\mathrm{dB} \text {, mean } \pm \mathrm{SD})\end{array}$} & \multicolumn{3}{|c|}{ P Values } \\
\hline & & $P_{1}$ & $P_{2}$ & $P_{3}$ & & $\mathrm{P}_{4}$ & $P_{5}$ & $P_{6}$ \\
\hline Pre-treatment & $68.33 \pm 26.19$ & 0.004 & 0.008 & 0.105 & $62.48 \pm 25.11$ & $<0.001$ & $<0.001$ & $<0.001$ \\
\hline Two Weeks after Treatment & $57.71 \pm 31.08$ & & & & $42.86 \pm 24.87$ & & & \\
\hline Four Weeks after Treatment & $44.86 \pm 25.69$ & & & & $30.34 \pm 22.93$ & & & \\
\hline
\end{tabular}

CIMT, constraint-induced music therapy; $\mathrm{dB}$, decibels; SD, standard deviation. $\mathrm{P}_{1}$ refers to the difference between results obtained before treatment and two weeks after treatment in the music therapy group; $P_{2}$ refers to the difference between results obtained before treatment and four weeks after treatment in the music therapy group; $P_{3}$ refers to the difference between results obtained at two weeks after treatment and those obtained at four weeks after treatment in the music therapy group; $P_{4}$ refers to the difference between results obtained before treatment and two weeks after treatment in the non-music therapy group; $P_{5}$ refers to the difference between results obtained before treatment and four weeks after treatment in the non-music therapy group; $\mathrm{P}_{6}$ refers to the difference between results obtained at two weeks after treatment and those obtained at four weeks after treatment in the non-music therapy group.

Table 3. Inter-Group Comparison of Hearing Thresholds Between CIMT and Non-CIMT Groups

\begin{tabular}{llll}
\hline Time Point & CIMT & Cases & Hearing Level (dB, mean \pm SD) \\
\hline Pre-treatment & Yes & 9 & $68.33 \pm 26.19$ \\
\cline { 2 - 4 } & No & 35 & $62.48 \pm 25.11$ \\
\hline Two Weeks after Treatment & Yes & 9 & $57.71 \pm 31.08$ \\
\cline { 2 - 4 } & No & 35 & $42.86 \pm 24.87$ \\
\hline Four Weeks after Treatment & Yes & 9 & $44.86 \pm 25.69$ \\
& No & 35 & $30.34 \pm 22.93$
\end{tabular}

CIMT, constraint-induced music therapy; $\mathrm{dB}$, decibels; SD, standard deviation.

Table 4. Comparison of Interaural Hearing Gap Between CIMT and Non-CIMT Groups

\begin{tabular}{llll}
\hline Time Point & CIMT & Cases & Interaural Hearing Gap (dB, mean \pm SD) \\
\hline Pre-treatment & Yes & 9 & $38.40 \pm 24.48$ \\
& No & 35 & $40.86 \pm 21.50$ \\
\hline Two Weeks after Treatment & Yes & 9 & $29.86 \pm 29.56$ \\
\cline { 2 - 4 } & No & 35 & $24.43 \pm 22.00$ \\
\hline Four Weeks after Treatment & Yes & 9 & $26.53 \pm 25.95$ \\
\cline { 2 - 4 } & No & 35 & $19.27 \pm 19.76$
\end{tabular}

CIMT, constraint-induced music therapy; dB, decibels; SD, standard deviation.

Table 5. Comparison of Hearing Recovering Rate Between CIMT and Non-CIMT Groups

\begin{tabular}{llll}
\hline Time Point & CIMT & Cases & Recovering Rate (\%) $)^{*}$ \\
\hline Two Weeks after Treatment & Yes & 9 & 41.91 \\
\cline { 2 - 4 } & No & 35 & 66.02 \\
\hline Four Weeks after Treatment & Yes & 9 & 46.71 \\
\cline { 2 - 4 } & No & 35 & 86.95 \\
\hline
\end{tabular}

${ }^{*}$ Hearing recovering rate $=$ (pre-treatment AC of the affected ear - post-treatment AC of the affected ear) / (pre-treatment AC of the affected ear - AC of the intact ear). AC, air conduction hearing threshold; CIMT, constraint-induced music therapy. 


\begin{tabular}{|c|c|c|c|c|c|c|c|c|}
\hline \multirow[t]{2}{*}{ Variable } & \multicolumn{4}{|c|}{ Two Weeks Post-treatment } & \multicolumn{4}{|c|}{ Four Weeks Post-treatment } \\
\hline & Cases & No. of HR (\%) & OR $(95 \% \mathrm{CI})$ & $\mathrm{P}$ & Cases & No. of HR (\%) & OR $(95 \% \mathrm{CI})$ & $P$ \\
\hline \multicolumn{9}{|l|}{ CIMT } \\
\hline Yes & 9 & $1(11)$ & 1 (ref) & & 9 & $2(22)$ & 1 (ref) & \\
\hline No & 35 & $12(34)$ & $3.84(0.18-81.65)$ & 0.388 & 35 & $20(57)$ & $2.70(0.15-47.60)$ & 0.497 \\
\hline \multicolumn{9}{|c|}{ Pre-treatment Hearing Level } \\
\hline$\leq 60 \mathrm{~dB}$ & 19 & $12(63)$ & $125(4.88-3181)$ & 0.004 & 19 & $16(84)$ & $45.41(3.60-573)$ & 0.003 \\
\hline$>60 \mathrm{~dB}$ & 25 & $1(4)$ & 1 (ref) & & 25 & $6(24)$ & 1 (ref) & \\
\hline \multicolumn{9}{|l|}{ Age } \\
\hline$\leq 50$ years & 16 & $8(50)$ & $20.58(1.141-371)$ & 0.040 & 16 & $11(69)$ & $6.40(0.72-56.49)$ & 0.095 \\
\hline$>50$ years & 28 & $5(5)$ & 1 (ref) & & 28 & $11(39)$ & 1 (ref) & \\
\hline \multicolumn{9}{|l|}{ Gender } \\
\hline Male & 23 & $7(34)$ & $1.40(0.18-11.29)$ & 0.750 & 23 & $13(57)$ & $1.80(0.23-13.84)$ & 0.572 \\
\hline Female & 21 & $6(29)$ & 1 (ref) & & 21 & $9(43)$ & 1 (ref) & \\
\hline \multicolumn{9}{|l|}{ Side of Ear } \\
\hline Right & 22 & $4(18)$ & 1 (ref) & & 22 & $5(23)$ & 1 (ref) & \\
\hline Left & 22 & $9(41)$ & $4.54(0.38-54.71)$ & 0.233 & 22 & $17(77)$ & $33.82(2.72-421)$ & 0.006 \\
\hline
\end{tabular}

*Hearing recovery is defined as hearing threshold $\leq 25 \mathrm{~dB}$. Cl, confidence interval; CIMT, constraint-induced music therapy; dB, decibel; HR, hearing recovery; OR, odds ratio; Ref, reference.

2 left ears. The mean age of CIMT patients was 57.44 years $(P=0.346)$. None of the patients who received CIMT presented complications or side effects, and all of the CIMT participants described it as an enjoyable therapeutic addition that helped relieve the stress and anxiety associated with SSNHL. The 35 patients in the non-CIMT retrospective group included 15 females/20 males involving 15 right ears and 20 left ears. The mean age of non-CIMT patients was 51.23 years $(P=0.346)$.

We selected $60 \mathrm{~dB}$ as the cutoff pre-treatment hearing level, because the mean hearing threshold of all participants prior to treatment was $63.68 \mathrm{~dB}$. An age threshold of 50 was adopted for the classification of patients as older or younger, because the mean age of the participants was 52.5 years. As shown in Table 1, the CIMT and non-CIMT groups were comparable in terms of pre-treatment hearing level $(P=0.710)$, age $(P=0.124)$, gender $(P=0.272)$, and side of ear $(P=0.132)$.

Table 2 lists an intra-group comparison of audiometric results from patients in the CIMT and non-CIMT groups. In the CIMT group, the mean post-treatment hearing threshold at two weeks post-treatment (57.71 \pm $31.08 \mathrm{~dB})$ and four weeks post-treatment $(44.86 \pm 25.69 \mathrm{~dB})$ was significantly better than before treatment $(68.33 \pm 26.19 \mathrm{~dB} ; \mathrm{P}=0.004$ and 0.008 , respectively). However, we did not observe a significant difference between the mean hearing thresholds at two weeks and four weeks post-treatment
$(P=0.105)$. In non-CIMT group, the mean post-operative hearing thresholds were significantly better at two weeks post-treatment $(42.86 \pm 24.87 \mathrm{~dB})$ and four weeks post-treatment $(30.34 \pm 22.93 \mathrm{~dB}$ ) than they were pre-operatively $(62.48 \pm 25.11 \mathrm{~dB}$, both $\mathrm{P}<0.001)$. The mean hearing threshold at four weeks post-treatment $(30.34 \pm 22.93 \mathrm{~dB})$ also presented a significant improvement over the hearing threshold at two weeks post-treatment $(42.86 \pm 24.87 \mathrm{~dB}$, $\mathrm{P}<0.001)$.

Table 3 presents an inter-group comparison of audiometric results between the CIMT and non-CIMT groups. The two groups were comparable in terms of mean pre-treatment hearing threshold $(68.33 \pm 26.19 \mathrm{~dB}$ in CIMT group and $62.48 \pm 25.11 \mathrm{~dB}$ in non-CIMT group, $\mathrm{P}=0.531$ ). No statistically significant differences were observed between the two groups with regard to the mean hearing thresholds at two weeks post-treatment $(57.71 \pm 31.08 \mathrm{~dB}$ in CIMT group and $42.86 \pm 24.87 \mathrm{~dB}$ in non-CIMT group, $\mathrm{P}=0.167)$ and four weeks post-treatment $(44.86 \pm 25.69 \mathrm{~dB}$ in CIMT group and $30.34 \pm 22.93 \mathrm{~dB}$ in non-CIMT group, $\mathrm{P}=0.067)$.

Table 4 lists an inter-group comparison of differences in interaural hearing threshold between the CIMT and non-CIMT Groups. The two groups were comparable prior to treatment (interaural hearing gap $38.40 \pm 24.48 \mathrm{~dB}$ in CIMT group and $40.86 \pm 21.50 \mathrm{~dB}$ in non-CIMT group, $\mathrm{P}=0.780$ ). After treatment, we were unable to detect statistically significant differences in interau-

\begin{tabular}{|c|c|c|c|c|c|c|}
\hline Variables & Amplitude $(\mu \mathrm{V})$ & Latency (ms) & $\mathrm{P}_{1}$ & $\mathrm{P}_{2}$ & $\mathrm{P}_{3}$ & $\mathrm{P}_{4}$ \\
\hline \multicolumn{3}{|l|}{ Pre-treatment } & \multirow[t]{6}{*}{0.063} & \multirow[t]{6}{*}{0.031} & \multirow[t]{6}{*}{0.094} & \multirow[t]{6}{*}{0.063} \\
\hline Affected ear (mean \pm SD) & $2.10 \pm 0.55$ & $342.63 \pm 20.49$ & & & & \\
\hline Healthy ear (mean \pm SD) & $1.75 \pm 0.92$ & $355.00 \pm 21.01$ & & & & \\
\hline \multicolumn{3}{|l|}{ Four Weeks Post-treatment } & & & & \\
\hline Affected ear (mean $\pm S D)$ & $2.42 \pm 1.44$ & $347.00 \pm 22.71$ & & & & \\
\hline Healthy ear (mean $\pm S D$ ) & $3.19 \pm 1.77$ & $342.75 \pm 42.12$ & & & & \\
\hline
\end{tabular}

$P_{1}$ refers to the difference in amplitude in affected ears before treatment and at four weeks after treatment; $P_{2}$ refers to the difference in amplitude in the healthy ears before treatment and at four weeks after treatment; $P_{3}$ refers to the difference in latency in affected ears before treatment and at four weeks after treatment; $P_{4}$ refers to the difference in latency in healthy ears before treatment and at four weeks after treatment. $\mu \mathrm{V}$, microvolt; $\mathrm{ms}$, millisecond. 
ral hearing gap between the two groups at two weeks post-treatment $(29.86 \pm$ $29.56 \mathrm{~dB}$ in CIMT group and $24.43 \pm 22.00 \mathrm{~dB}$ in non-CIMT group, $\mathrm{P}=0.802$ ) or four weeks post-treatment $(26.53 \pm 25.95 \mathrm{~dB}$ in CIMT group and $19.27 \pm 19.76$ $\mathrm{dB}$ in non-CIMT group, $\mathrm{P}=0.611$ )

Table 5 presents a comparison of hearing recovering rates between the CIMT and non-CIMT groups. No statistically significant differences were observed in the hearing recovering rate between the two groups at two weeks post-treatment $(41.91 \%$ in CIMT group and $66.02 \%$ in non-CIMT group, $\mathrm{P}=$ 0.478 ) or four weeks post-treatment $(46.71 \%$ in CIMT group and $88.95 \%$ in non-CIMT group, $\mathrm{P}=0.217$ ).

Table 6 lists the results of multivariate logistic regression used to identify potential predictors of hearing recovery at two and four weeks post-treatment. This analysis revealed no statistically significant correlations between post-treatment hearing recovery and the application of CIMT (odds ratio, OR, 3.84, confidence interval, $\mathrm{Cl}, 0.18-81.65, \mathrm{P}=0.388$ at two weeks post-treat ment; and $\mathrm{OR}=2.70, \mathrm{Cl}=0.15-47.60, \mathrm{P}=0.497$ at four weeks post-treatment) It was observed that patients with better pre-treatment hearing level ( $\leq 60 \mathrm{~dB})$ were more likely than those with worse hearing $(>60 \mathrm{~dB})$ to present hearing recovery at two weeks $(\mathrm{OR}=125, \mathrm{Cl}=4.88-3181, \mathrm{P}=0.004)$ and four weeks $(\mathrm{OR}=45.41, \mathrm{Cl}=3.60-573, \mathrm{P}=0.003)$ post-treatment. The other three factors (age, gender, and side of ear) did not have a significant influence on hearing recovery at two or four weeks post-treatment.

Table 7 presents a comparison of the amplitude and latency values of the P300 component in each ear before and after treatment. We succeeded in recording the $\mathrm{P} 300$ component in $66.7 \%$ of the subjects ( 6 of 9 patients). There was a significant difference in amplitude value in the healthy ears before treatment $(1.75 \pm 0.92 \mu \mathrm{V})$ and at four weeks after treatment $(3.19 \pm 1.77 \mu \mathrm{V}, \mathrm{P}=$ 0.031 ). We observed no other statistically significant differences in P300 amplitudes or latencies in the affected or healthy ears (all P > 0.05, Table 7)

\section{DISCUSSION}

\section{Main Findings}

In both the CIMT and non-CIMT groups, we observed a significant improvement in hearing thresholds at two weeks and four weeks post-treatment ( $\mathrm{Ta}$ ble 2). Nevertheless, we failed to detect any statistically significant differences between CIMT and non-CIMT groups in terms of hearing recovery when comparing outcome variables, including hearing threshold (Table 3), interaural hearing gap (Table 4), and hearing recovering rate (Table 5 ). Patients who underwent CIMT in conjunction with conventional steroid therapy were no more likely than patients who received only steroid therapy to recover hearing to normal limits (Table 6). In a subgroup assessment of 6 patients, the long-latency auditory evoked potential did not provide any significant indications of neuroplasticity in response to CIMT (Table 7).

\section{New Therapeutic Direction for SSNHL: Neuroplasticity}

One set of clinical practice guidelines for SSNHL in the US advises against the routine prescription of antivirals, thrombolytics, vasodilators, vasoactive substances, or antioxidants for patients with SSNHL. Furthermore, the benefit/harm balance for steroid therapy remains uncertain [1]. Considering the devastating effects that SSNHL can have on the patient's quality of life, devising a new therapeutic direction to deal with SSNHL is no doubt a worthy endeavor [18].

Neuroplasticity can be viewed as adaptive responses to conditions associated with a behavioral gain, such as learning. Advances in our understanding of brain plasticity have led to the development of promising interventions for several neurological conditions and disorders. These methods are meant to promote adaptive neuroplastic changes to compensate for lost functions or to maximize remaining functions [43-45]. Results from numerous human and animal studies indicate that there are strong links between the expenditure of effort in training and use-dependent structural adaptation in the brain [4650]. Plastic changes in brain structure contribute to an adaptive gain in func- tion in healthy individuals (e.g., learning skills or creating memories) as well as recovery from brain damage. Cortical reorganization has been shown to compensate for loss of function or increase residual functions following brain lesion. Neuroplasticity can relieve initial deficits in behavior as well as perceptual and cognitive skills [51]. Plasticity-promoting theory has been implicated to achieve clinical gains and improve behavioral outcomes in parallel with increased brain plasticity. Several interventions have been developed to enhance brain plasticity, such as Hebbian learning [52], task-specific training [53], transcranial magnetic stimulation [54], deep brain stimulation [55], cognitive behavioral therapy [56], physical training [57], and neuropharmacotherapies that involve the molecular manipulation of numerous cellular and synaptic pathways [19,58,59].

Adaptive plasticity refers to a neural change in a positive direction for behavioral gain (e.g., skill learning) or functional compensation (e.g., poststroke recovery). However, other forms of plasticity can induce maladaptive cortical reorganization, such as focal hand dystonia [30], phantom limb pain [60], and tinnitus [61,62]. These can have a negative effect on disease pathogenesis, resulting in unanticipated and undesired consequences [19,22,51,63-65]. Dealing with these adverse consequences requires interventions aimed at blocking or hindering plasticity, such as behavioral training to overcome focal hand dystonia [30], phantom limb pain [60], and tinnitus [61].

\section{Additive Effect of CIMT on Hearing Recovery}

It is possible that gains in functional recovery could be enhanced by preventing maladaptive plastic changes [18], and a promising therapeutic start may indicate a new direction for the management of SSNHL.

Based on previous findings, it is reasonable to expect spontaneous recovery in a considerable proportion of patients (32\% to $65 \%)[2,5,14]$. However, many patients face permanent hearing deficit, which brings on frustration, anxiety, insecurity, loneliness, depression, and social isolation $[1,66]$. This treatment limitation and potentially serious consequences highlight the need to aggressively explore a new avenue for therapeutic approach.

In animal experiments, researchers observed that a decrease in the spontaneous and driven firing rate in auditory nerve fibers following acoustic trauma commonly triggers central reorganization [26,27,67]. Researchers have also discovered that animals that undergo post-traumatic acoustic stimulation are less affected by hearing loss associated with hair cell damage to the cochlea, compared to animals that do not undergo such stimulation $[23,24,26]$. In other words, acoustic energy delivered to ciliated cells in the cochlea can be converted into electrical impulses that are transmitted to the auditory cortex through auditory nerves [68]. It has been surmised that acoustic stimulation (1) compensates for a loss of afferent neural inputs and (2) prevents maladaptive neuroplasticity in the brain. Both of these effects could promote hearing recovery [24,25].

In human studies using functional magnetic resonance imaging (fMRI) $[69,70]$, many patients with SSNHL present an altered auditory cortical response under auditory stimulation. Magnetoencephalographic (MEG) studies have also revealed immediate and protracted changes in the function of auditory pathways among patients with SSNHL [71-75]. Overall, human studies support the findings of animal studies pertaining to SSNHL-induced brain plasticity.

In 2012, López-González et al. compared the outcomes of 65 SSNHL patients treated with medications (steroids, piracetam, and antioxidants) and 67 patients treated with medication as well as sound therapy (a combination of music and speech). They reported much higher hearing recovery among patients that underwent stimulation via sound therapy, compared to those without acoustic stimulation [68]. However, that study was based solely on audiometric outcomes. The absence of neuroimaging results (e.g., fMRI and MEG) precludes an examination of the extent and degree of plastic changes in the brain in response to SSNHL.

In 2014, Okamoto et al. [22] adopted a neuro-rehabilitation approach well-established in treating stroke patients (constraint therapy or con- 
straint-induced movement therapy) in an attempt to enhance hearing recovery in patients with SSNHL [76]. All 53 of the SSNHL patients received traditional corticosteroid therapy, 22 of whom were also administered CIMT. They reported that the interaural hearing gap in the CIMT group was significantly smaller than that seen in the non-CIMT group between 1 and 6 months after treatment. In the current study, the interaural hearing gap in the CIMT and non-CIMT groups was smaller at two and four weeks after treatment. However, we did not observe statistically significant differences in interaural hearing gaps between the two groups (Table 4).

In both groups, we observed a significant improvement in hearing thresholds at two weeks and four weeks post-treatment (Table 2); however, there were no statistically significant differences in post-treatment hearing thresholds between the two groups (Table 3). This outcome was confirmed by multivariate analysis, which failed to detect a predictive association between the application of CIMT and hearing recovery to normal limits (Table 6). Okamoto et al. [22] did not compare post-treatment hearing thresholds between CIMT and non-CIMT groups.

We also compared outcomes in the two groups in terms of hearing recovering rate (Table 5). No significant intra-group differences were observed in hearing recovering rate. In 2015, Liu et al. [41] obtained similar results between an experimental group (47 patients receiving methylprednisolone and relaxing music) and a control group (42 patients receiving only methylprednisolone). Nonetheless, Liu et al. reported that acoustic treatment combined with steroid therapy alleviated the symptoms of SSNHL-related anxiety. In the current study, all 9 of the patients in the CIMT group reported a reduction in the stress and anxiety associated with SSNHL to tolerable levels.

Our findings do not provide audiometric evidence of an additive effect of CIMT on hearing recovery; however, we were able to demonstrate a beneficiary effect on the relief of stress and anxiety.

\section{Degree of Neuroplasticity}

Okamoto et al. [22] selectively included 6 patients in a CIMT subgroup for the analysis of neural activity using MEG, including an $\mathrm{N} 1 \mathrm{~m}$ response (generated mainly in the belt and parabelt areas of auditory cortex) [77] and auditory steady state response (ASSR, generated in the primary auditory cortex) [78]. Their results demonstrated a contralateral dominance of neural activity in both the primary auditory cortex (ASSR) and auditory belt areas $(\mathrm{N} 1 \mathrm{~m})$ following steroid treatment in conjunction with CIMT. Dominance in the contralateral hemisphere is generally also found in healthy subjects with normal hearing $[7,75]$. Okamoto et al. claimed that the contralateral dominance of neural activity was an indication that SSNHL-induced contralateral cortical maladaptive plastic changes had been reversed. However, it may be premature to assert an association between hearing recovery and the reversal of cortical reorganization. Furthermore, audiometric outcome data of the 6 patients were not included in their report.

In the current study, we investigated post-treatment neuroplasticity by measuring the P300 component of long-latency auditory evoked potential. P300 is seen as a promising assessment tool for the detection of central changes in patients with sensorineural hearing loss [79-81]. We succeeded in recording the P300 component in 6 of the 9 patients studied (66.7\%), which is consistent with the rates reported in the literature [80]. We observed a statistically significant difference in the amplitude values obtained from healthy ears before treatment and four weeks after treatment $(P=$ 0.031 , Table 7). However, when comparing the amplitudes and latencies of the affected ears (before treatment and four weeks after treatment), no significant changes were found (both $P>0.05$ ). Our electrophysiological findings are not in agreement with those reported by Okamoto et al. [22]. Nevertheless, we must not jump to any conclusions regarding the neuroplastic effects of CIMT on hearing recovery in patients with SSNHL. The sample sizes in the current study ( 6 patients) and in the study by Okamoto et al. (6 patients) [22] were of insufficient size to avoid bias in our sampling. A large number of patients will be required to determine the true degree of neuroplasticity in patients receiving CIMT.

\section{Study Limitations}

To the best of our knowledge, this was only the second study aimed at validating assertions that CIMT can prevent or reverse SSNHL-induced maladaptive cortical reorganization, thereby potentiating subsequent hearing recovery. Nonetheless, the current study was subject to a number of limitations. First, a larger sample size will be required to avoid sampling bias. Second, we acknowledge that bias may have been introduced at any stage of the study, due to the inclusion of a retrospective study arm. Selection bias (e.g., a non-representative study population) and information bias (e.g., imprecise measurements or incorrect recording of outcomes) could be avoided by adopting a double-blind, randomized, placebo-controlled trial in the future. All patients in the study received current first-line treatment (i.e., steroid therapy); therefore, it is unlikely that the functional recovery can be attributed solely to CIMT. Steroid therapy of this type has been shown to have success rates of 50 to $80 \%$ [11]. Outcome analysis was further complicated by spontaneous recovery, which has been reported in $32 \%$ to $65 \%$ of all cases of SSNHL $[2,5,14]$. Thus, it would be very difficult to determine the degree to which CIMT treatment exceeds spontaneous recovery. According to the literature, spontaneous improvement in hearing occurs primarily within two weeks after onset, and late recovery is highly unlikely for patients who do not undergo treatment. In the future, researchers should recruit volunteer patients who maintain the same level of hearing loss and who do not receive treatment for a period of two weeks following diagnosis with idiopathic SSNHL. A delayed treatment group of this sort could help to clarify the true effectiveness of CIMT on hearing recovery by eliminating the confounding effects of spontaneous recovery.

\section{CONCLUSIONS}

This study confirms that CIMT is a safe and cost-effective addition to corticosteroid treatment for SSNHL; however, our preliminary results could neither identify the additive effects of CIMT on hearing recovery nor confirm the degree of neuroplasticity in patients with SSNHL. Nevertheless, it appears that CIMT provides an enjoyable adjunct to conventional steroid treatment for the relief of stress and anxiety associated with SSNHL.

\section{ARTICLE INFORMATION}

*Correspondence: Chin-Lung Kuo, MD, PhD, Department of Otolaryngology, Taoyuan Armed Forces General Hospital, Taoyuan, Taiwan. E-mail: drkuochinlung@gmail.com

Received: Nov. 11, 2018; Accepted: Dec. 16, 2018; Published: Apr. 10, 2019

DOI: 10.24983/scitemed.nnr.2019.00110

Acknowledgments: Dr. Kuo thanks Mr. Yi Chen for his assistance in completion of audiometric and electrophysiological examinations.

Ethics Approval and Consent to Participate: The study is in accordance with the ethical standards of the 1964 Helsinki declaration and its later amendments or comparable ethical standards.

Funding: This study was sponsored by grants from the Medical Affairs Bureau Ministry of National Defense (MAB-107099) and Taoyuan Armed Forces General Hospital (AFTYGH-10734).

Conflict of Interest: The authors report no financial or other conflict of interest relevant to this article, which is the intellectual property of the authors.

Copyright @ 2019 The Author. This is an open-access article distributed under the terms of the Creative Commons Attribution 4.0 International License (CC-BY). 


\section{REFERENCES}

1. Stachler RJ, Chandrasekhar SS, Archer SM, et al. Clinical practice guideline: Sudden hearing loss. Otolaryngology--head and neck surgery: official journal of American Academy of Otolaryngology-Head and Neck Surgery 2012;146(3 Sup$\mathrm{pl}):$ S1-35.

2. Mattox DE, Simmons FB. Natural history of sudden sensorineural hearing loss. The Annals of otology, rhinology, and laryngology 1977;86(4 Pt 1):463-480.

3. Klemm E, Deutscher A, Mosges R. [a present investigation of the epidemiology in idiopathic sudden sensorineural hearing loss]. Laryngo- rhino- otologie 2009;88(8):524-527.

4. Rauch SD, Halpin CF, Antonelli PJ, et al. Oral vs intratympanic corticosteroid therapy for idiopathic sudden sensorineural hearing loss: A randomized trial. Jama 2011;305(20):2071-2079.

5. Byl FM, Jr. Sudden hearing loss: Eight years' experience and suggested prog nostic table. The Laryngoscope 1984;94(5 Pt 1):647-661.

6. Wu CS, Lin HC, Chao PZ. Sudden sensorineural hearing loss: Evidence from taiwan. Audiology \& neuro-otology 2006;11(3):151-156.

7. Li LP, Shiao AS, Chen LF, et al. Healthy-side dominance of middle- and long-la tency neuromagnetic fields in idiopathic sudden sensorineural hearing loss. The European journal of neuroscience 2006;24(3):937-946.

8. Saunders JE, Luxford WM, Devgan KK, Fetterman BL. Sudden hearing loss in acoustic neuroma patients. Otolaryngology--head and neck surgery : official journal of American Academy of Otolaryngology-Head and Neck Surgery 1995; 113(1):23-31.

9. Guyot JP, Thielen K. [evolution of sudden deafness without treatment]. Schweizerische medizinische Wochenschrift Supplementum 2000;116:93S-96S.

10. Wilson WR, Byl FM, Laird N. The efficacy of steroids in the treatment of idiopathic sudden hearing loss. A double-blind clinical study. Archives of otolaryngology 1980;106(12):772-776.

11. Ferri E, Frisina A, Fasson AC, Armato E, Spinato G, Amadori M. Intratympanic steroid treatment for idiopathic sudden sensorineural hearing loss after failure of intravenous therapy. ISRN otolaryngology 2012;2012:647271.

12. Wei BP, Stathopoulos D, O'Leary S. Steroids for idiopathic sudden sensorineural hearing loss. The Cochrane database of systematic reviews 2013;7:CD003998.

13. Conlin $A E$, Parnes LS. Treatment of sudden sensorineural hearing loss: I. A systematic review. Archives of otolaryngology--head \& neck surgery 2007;133(6):573-581.

14. Conlin AE, Parnes LS. Treatment of sudden sensorineural hearing loss: li. A meta-analysis. Archives of otolaryngology--head \& neck surgery 2007;133(6):582586.

15. Labus J, Breil J, Stutzer H, Michel O. Meta-analysis for the effect of medical therapy vs. Placebo on recovery of idiopathic sudden hearing loss. The Laryngoscope 2010;120(9):1863-1871.

16. Wei BP, Stathopoulos D, O'Leary S. Steroids for idiopathic sudden sensorineural hearing loss. The Cochrane database of systematic reviews 2013;7(7):CD003998.

17. Liu D, Ahmet A, Ward L, et al. A practical guide to the monitoring and management of the complications of systemic corticosteroid therapy. Allergy, asthma, and clinical immunology : official journal of the Canadian Society of Allergy and Clinical Immunology 2013;9(1):30.

18. Kuo CL. Neuroplasticity-targeted intervention for idiopathic sudden sensorineural hearing loss: A new therapeutic direction. Neurology and Neuroscience Research 2017;1(1):1-5.

19. Cramer SC, Sur M, Dobkin BH, et al. Harnessing neuroplasticity for clinical applications. Brain : a journal of neurology 2011;134(Pt 6):1591-1609.

20. Fuchs E, Flugge G. Adult neuroplasticity: More than 40 years of research. Neural plasticity 2014;2014:541870.

21. Pascual-Leone A, Amedi A, Fregni F, Merabet LB. The plastic human brain cortex. Annual review of neuroscience 2005;28:377-401.

22. Okamoto $\mathrm{H}$, Fukushima M, Teismann $\mathrm{H}$, et al. Constraint-induced sound therapy for sudden sensorineural hearing loss--behavioral and neurophysiological outcomes. Scientific reports 2014;4:3927.

23. Fukushima N, White $\mathrm{P}$, Harrison RV. Influence of acoustic deprivation on re covery of hair cells after acoustic trauma. Hearing research 1990;50(1-2):107118.

24. Niu X, Tahera Y, Canlon B. Protection against acoustic trauma by forward and backward sound conditioning. Audiology \& neuro-otology 2004;9(5):265-273.

25. Willott JF, Bross L. Effects of prolonged exposure to an augmented acoustic environment on the auditory system of middle-aged c57bl/6j mice: Cochlear and central histology and sex differences. The Journal of comparative neurology 2004;472(3):358-370.

26. Norena AJ, Eggermont JJ. Enriched acoustic environment after noise trauma reduces hearing loss and prevents cortical map reorganization. The Journal of neuroscience : the official journal of the Society for Neuroscience 2005;25(3):699705.

27. Rajan R, Irvine DR, Wise LZ, Heil P. Effect of unilateral partial cochlear lesions in adult cats on the representation of lesioned and unlesioned cochleas in primary auditory cortex. The Journal of comparative neurology 1993;338(1):1749.

28. Blanton S, Wolf SL. An application of upper-extremity constraint-induced movement therapy in a patient with subacute stroke. Phys Ther 1999;79(9):847-853.

29. Dromerick AW, Edwards DF, Hahn M. Does the application of constraint-induced movement therapy during acute rehabilitation reduce arm impairment after ischemic stroke? Stroke; a journal of cerebral circulation 2000;31(12):2984-2988.

30. Candia V, Elbert T, Altenmuller E, Rau H, Schafer T, Taub E. Constraint-induced movement therapy for focal hand dystonia in musicians. Lancet 1999;353(9146):42.

31. Stock R, Thrane G, Anke A, Gjone R, Askim T. Early versus late-applied constraint-induced movement therapy: A multisite, randomized controlled trial with a 12-month follow-up. Physiother Res Int 2018;23(1).

32. Simon-Martinez C, Mailleux L, Ortibus E, et al. Combining constraint-induced movement therapy and action-observation training in children with unilateral cerebral palsy: A randomized controlled trial. BMC Pediatr 2018;18(1):250.

33. Shikako-Thomas K, Fehlings D, Germain M, Gordon AM, Maynard D, Majnemer A. Current practice "constraints" in the uptake and use of intensive upper extremity training: A canadian perspective. Phys Occup Ther Pediatr 2018;38(2):143-156.

34. Okabe N, Himi N, Nakamura-Maruyama E, et al. Constraint-induced movement therapy improves efficacy of task-specific training after severe cortical stroke depending on the ipsilesional corticospinal projections. Experimental neurology 2018;305:108-120.

35. Shepard $\mathrm{TH}$, Brent RL, Friedman JM, et al. Update on new developments in the study of human teratogens. Teratology 2002;65(4):153-161.

36. Clement S, Braithwaite SS, Magee MF, et al. Management of diabetes and hyperglycemia in hospitals. Diabetes Care 2004;27(2):553-591.

37. Rivner $\mathrm{MH}$. Steroid treatment for myasthenia gravis: Steroids are overutilized. Muscle Nerve 2002;25(1):115-117.

38. Fung MA, Berger TG. A prospective study of acute-onset steroid acne associated with administration of intravenous corticosteroids. Dermatology 2000;200(1):43-44

39. Gstoettner W, Neuwirth-Riedl K, Swoboda H, Mostbeck W, Burian M. Specificity of auditory brainstem response audiometry criteria in acoustic neuroma screening as a function of deviations of reference values in patients with cochlear hearing loss. European archives of oto-rhino-laryngology : official journal of the European Federation of Oto-Rhino-Laryngological Societies 1992;249(5):253-256

40. Helland PB. Calm water piano. Soothing Relaxation. Available at: https://soothingrelaxation.bandcamp.com. Acessed November 17, 2018.

41. Liu Z, Liang Y, Yang C, et al. [the clinical efficacy of composite acoustic therapy in patients of sudden deafness with tinnitus]. Lin Chung Er Bi Yan Hou Tou Jing Wai Ke Za Zhi 2015;29(4):330-333.

42. Carhart R, Jerger JF. Preferred method for clinical determination of pure-tone thresholds. Journal of Speech and Hearing Disorders 1959;24(4):330-345.

43. Woollett K, Spiers HJ, Maguire EA. Talent in the taxi: A model system for exploring expertise. Philosophical transactions of the Royal Society of London Series $B$, Biological sciences 2009;364(1522):1407-1416.

44. Bird CM, Burgess N. The hippocampus and memory: Insights from spatial processing. Nature reviews Neuroscience 2008;9(3):182-194.

45. Gaser C, Schlaug G. Brain structures differ between musicians and non-musicians. The Journal of neuroscience : the official journal of the Society for Neuroscience 2003;23(27):9240-9245.

46. Schneider P, Scherg M, Dosch HG, Specht HJ, Gutschalk A, Rupp A. Morphology of heschl's gyrus reflects enhanced activation in the auditory cortex of 
musicians. Nature neuroscience 2002;5(7):688-694.

47. Pantev C, Roberts LE, Schulz M, Engelien A, Ross B. Timbre-specific enhancement of auditory cortical representations in musicians. Neuroreport 2001;12(1):169-174.

48. Mechelli A, Crinion JT, Noppeney U, et al. Neurolinguistics: Structural plasticity in the bilingual brain. Nature 2004;431(7010):757.

49. Anderson BJ, Eckburg PB, Relucio Kl. Alterations in the thickness of motor cortical subregions after motor-skill learning and exercise. Learning \& memory 2002;9(1):1-9.

50. Kleim JA, Lussnig E, Schwarz ER, Comery TA, Greenough WT. Synaptogenesis and fos expression in the motor cortex of the adult rat after motor skill learning. The Journal of neuroscience : the official journal of the Society for Neuroscience 1996;16(14):4529-4535.

51. Elbert T, Heim S. A light and a dark side. Nature 2001;411(6834):139.

52. Pennartz CM. Reinforcement learning by hebbian synapses with adaptive thresholds. Neuroscience 1997;81(2):303-319.

53. Hubbard IJ, Parsons MW, Neilson C, Carey LM. Task-specific training: Evidence for and translation to clinical practice. Occupational therapy international 2009;16(3-4):175-189.

54. Mantovani A, Aly M, Dagan Y, Allart A, Lisanby SH. Randomized sham controlled trial of repetitive transcranial magnetic stimulation to the dorsolateral prefrontal cortex for the treatment of panic disorder with comorbid major depression. Journal of affective disorders 2013;144(1-2):153-159.

55. Pepper J, Hariz M, Zrinzo L. Deep brain stimulation versus anterior capsulotomy for obsessive-compulsive disorder: A review of the literature. Journal of neurosurgery 2015;122(5):1028-1037.

56. Walkup JT, Albano AM, Piacentini J, et al. Cognitive behavioral therapy, sertraline, or a combination in childhood anxiety. The New England journal of medicine 2008;359(26):2753-2766.

57. Lo AC, Guarino PD, Richards LG, et al. Robot-assisted therapy for long-term upper-limb impairment after stroke. The New England journal of medicine 2010;362(19):1772-1783.

58. Vecsey CG, Hawk JD, Lattal KM, et al. Histone deacetylase inhibitors enhance memory and synaptic plasticity via creb:Cbp-dependent transcriptional activation. The Journal of neuroscience : the official journal of the Society for Neuroscience 2007;27(23):6128-6140.

59. Potter WB, O'Riordan KJ, Barnett D, et al. Metabolic regulation of neuronal plasticity by the energy sensor ampk. PloS one 2010;5(2):e8996.

60. Weiss T, Miltner WH, Adler T, Bruckner L, Taub E. Decrease in phantom limb pain associated with prosthesis-induced increased use of an amputation stump in humans. Neuroscience letters 1999;272(2):131-134.

61. Okamoto $\mathrm{H}$, Stracke $\mathrm{H}$, Stoll W, Pantev C. Listening to tailor-made notched music reduces tinnitus loudness and tinnitus-related auditory cortex activity. Proceedings of the National Academy of Sciences of the United States of America 2010;107(3):1207-1210.

62. Pantev $\mathrm{C}$, Okamoto $\mathrm{H}$, Teismann $\mathrm{H}$. Tinnitus: The dark side of the auditory cortex plasticity. Annals of the New York Academy of Sciences 2012;1252:253258.

63. Nudo RJ. Plasticity. NeuroRx : the journal of the American Society for Experimental NeuroTherapeutics 2006;3(4):420-427.
64. Bara-jimenez W, Catalan MJ, Hallett M, Gerloff C. Abnormal somatosensory homunculus in dystonia of the hand. Annals of neurology 1998;44(5):828-831.

65. Merzenich MM, Van Vleet TM, Nahum M. Brain plasticity-based therapeutics. Frontiers in human neuroscience 2014;8:385.

66. Mosges R, Koberlein J, Erdtracht B, Klingel R, Group R-IS. Quality of life in patients with idiopathic sudden hearing loss: Comparison of different therapies using the medical outcome short form (36) health survey questionnaire. Otology \& neurotology : official publication of the American Otological Society, American Neurotology Society [and] European Academy of Otology and Neurotology 2008;29(6):769-775.

67. Norena AJ, Eggermont JJ. Changes in spontaneous neural activity immediately after an acoustic trauma: Implications for neural correlates of tinnitus. Hearing research 2003;183(1-2):137-153.

68. Lopez-Gonzalez MA, Cambil E, Abrante A, Lopez-Fernandez R, Esteban F. [sound therapy in sudden deafness]. Acta otorrinolaringologica espanola 2012;63(3):165-172.

69. Suzuki M, Kouzaki H, Nishida Y, Shiino A, Ito R, Kitano H. Cortical representation of hearing restoration in patients with sudden deafness. Neuroreport 2002;13(14):1829-1832.

70. Bilecen D, Seifritz E, Radu EW, et al. Cortical reorganization after acute unilateral hearing loss traced by fmri. Neurology 2000;54(3):765-767.

71. Vasama JP, Makela JP, Pyykko I, Hari R. Abrupt unilateral deafness modifies function of human auditory pathways. Neuroreport 1995;6(7):961-964.

72. Vasama JP, Makela JP. Auditory pathway plasticity in adult humans after unilateral idiopathic sudden sensorineural hearing loss. Hearing research 1995;87(1-2):132-140.

73. Fujiki N, Naito $\mathrm{Y}$, Nagamine $\mathrm{T}$, et al. Influence of unilateral deafness on auditory evoked magnetic field. Neuroreport 1998;9(14):3129-3133.

74. Dietrich V, Nieschalk M, Stoll W, Rajan R, Pantev C. Cortical reorganization in patients with high frequency cochlear hearing loss. Hearing research 2001;158(1-2):95-101.

75. Po-Hung Li L, Shiao AS, Lin YY, et al. Healthy-side dominance of cortical neuromagnetic responses in sudden hearing loss. Annals of neurology 2003;53(6):810-815.

76. Taub E, Miller NE, Novack TA, et al. Technique to improve chronic motor deficit after stroke. Archives of physical medicine and rehabilitation 1993;74(4):347354.

77. Salminen NH, Tiitinen $\mathrm{H}$, Miettinen I, Alku P, May PJ. Asymmetrical representation of auditory space in human cortex. Brain research 2010;1306:93-99.

78. Pantev C, Roberts LE, Elbert T, Ross B, Wienbruch C. Tonotopic organization of the sources of human auditory steady-state responses. Hearing research 1996;101(1-2):62-74.

79. Didone DD, Garcia MV, Oppitz SJ, et al. Auditory evoked potential p300 in adults: Reference values. Einstein (Sao Paulo) 2016;14(2):208-212.

80. Reis AC, Frizzo AC, Isaac Mde L, Garcia CF, Funayama CA, Iorio MC. P300 in individuals with sensorineural hearing loss. Brazilian journal of otorhinolaryngology 2015;81(2):126-132.

81. Leite RA, Magliaro FCL, Raimundo JC, Bento RF, Matas CG. Monitoring auditory cortical plasticity in hearing aid users with long latency auditory evoked potentials: A longitudinal study. Clinics (Sao Paulo) 2018;73:e51. 